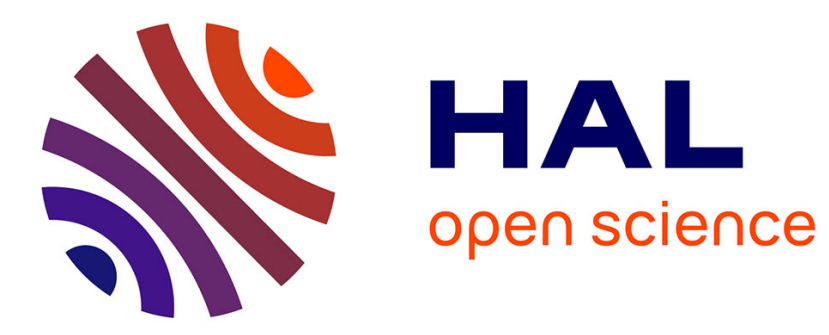

\title{
AH-Substructures in Neutrosophic Modules 1
}

\author{
Mohammad Abobala, Riad K Alhamido
}

\section{To cite this version:}

Mohammad Abobala, Riad K Alhamido. AH-Substructures in Neutrosophic Modules 1. International Journal of Neutrosophic Science, 2020, 7, pp.79 - 86. 10.5281/zenodo.3879702 . hal-02876526

\section{HAL Id: hal-02876526 \\ https://hal.science/hal-02876526}

Submitted on 10 Aug 2020

HAL is a multi-disciplinary open access archive for the deposit and dissemination of scientific research documents, whether they are published or not. The documents may come from teaching and research institutions in France or abroad, or from public or private research centers.
L'archive ouverte pluridisciplinaire HAL, est destinée au dépôt et à la diffusion de documents scientifiques de niveau recherche, publiés ou non, émanant des établissements d'enseignement et de recherche français ou étrangers, des laboratoires publics ou privés. 


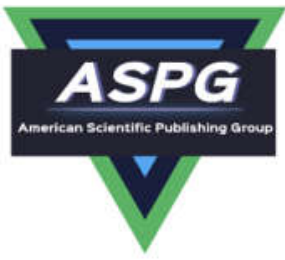

\title{
AH-Substructures in Neutrosophic Modules
}

\author{
${ }^{1}$ Mohammad Abobala and ${ }^{2}$ Riad K. Alhamido \\ ${ }^{1}$ Faculty of Science, Tishreen University, Lattakia, Syria \\ ${ }^{2}$ Faculty of Science, Alfurat University, Deir Elzor, Syria \\ 1e-mail: mohammadabobala777@gmail.com \\ 2e-mail: Riad-hamido1983@hotmail.com
}

\begin{abstract}
This article introduces the concept of AH-submodule, AHS-submodule of a neutrosophic module, and AHShomomorphism. This work presents some basic notions and properties of these concepts such as AH-Kernel, AHQuotient, and dimension, and determines the algebraic structure of weak neutrosophic module over a commutative ring $\mathrm{R}$.
\end{abstract}

Keywords: Neutrosophic module, AH-submodule, AHS-submodule, AH-Quotient.

\section{Introduction}

Neutrosophy as a new branch of philosophy founded by Smarandache has an interesting effect in real world problems, applications and algebraic studies. Recently, neutrosophic sets have been applied in the medical field such as diagnosis of bipolar disorder diseases [1], evaluation hospital medical care systems [2], intelligent medical decision support model based on soft computing and many other ares [3], and novel group decision making model for heart disease [4].

Many neutrosophic algebraic structures have been defined and studied such as neutrosophic rings, neutrosophic groups, neutrosophic vector spaces and refined neutrosophic rings. See[7, 8, 9, 10, 11, 12, 13, 14]. AH-substructures were defined for the first time in neutrosophic rings in [5], then they were introduced in refined neutrosophic rings in [2]. These structures have many symmetric properties which illustrate a line between classical algebra and neutrosophical algebra. AH-ideal in a neutrosophic ring $\mathrm{R}(\mathrm{I})$ is a set with form $P+Q I$ where $P, Q$ are ideals in R, other AH-structures can be defined to have many parts, each part has a special structure such (subspace, ideal, and submodule). AH-substructures were studied in refined neutrosophic rings, and neutrosophic vector spaces too [6,8]. In this paper we try to define AH-submodule and AHS-submodule of a neutrosophic module and introduce some of their elementary properties. Also, some interesting concepts were defined and applied in this study, such as neutrosophic module AHS-homomorphism, and AH-Quotient module.

\section{Motivation}

This work is an extension of studies about AH-substructures in neutrosophical algebraic structures in $[5,6,8]$.

\section{Preliminaries}

DOI: $10.5281 /$ zenodo.3879702

Received: March 08, 2020 Revised: April 18, 2020 Accepted: May 27, 2020 
In the following part, we recall some notions which will be used in our study.

Definition 2.1: [15]

Let $(\mathrm{N},+,$.$) be a module over the ring \mathrm{R}$. Then $(\mathrm{N}(\mathrm{I}),+,$.$) is called a weak neutrosophic module over the ring \mathrm{R}$, and it is called a strong neutrosophic module if it is a module over the neutrosophic ring $\mathrm{R}(\mathrm{I})$.

Here elements of $\mathrm{N}(\mathrm{I})$ have the form $\boldsymbol{x}+\boldsymbol{y} \boldsymbol{I} ; \boldsymbol{x}, \boldsymbol{y} \in \boldsymbol{N}$, i.e $\mathrm{N}(\mathrm{I})$ can be written as $\boldsymbol{N}(\boldsymbol{I})=\boldsymbol{N}+\boldsymbol{N} \boldsymbol{I}$.

Definition 2.2: [15]

Let $\mathrm{M}(\mathrm{I})$ be a strong neutrosophic module over the neutrosophic ring $\mathrm{R}(\mathrm{I})$ and $\mathrm{W}(\mathrm{I})$ be a nonempty set of $\mathrm{M}(\mathrm{I})$, then $\mathrm{W}(\mathrm{I})$ is called a strong neutrosophic submodule if W(I) itself is a strong neutrosophic module.

Definition 2.3: [15]

Let $\mathrm{U}(\mathrm{I})$ and $\mathrm{W}(\mathrm{I})$ be two strong neutrosophic modules and let $f: U(I) \rightarrow W(I)$, we say that $\mathrm{f}$ is a neutrosophic vector space homomorphism if

(a) $f(I)=I$.

(b) $f$ is a module homomorphism.

We define the kernel of $\mathrm{f}$ by $\operatorname{Ker}(\mathrm{f})=\{\mathrm{x} \in M(I) ; f(x)=0\}$.

Definition 2.4: [6]

Let R(I) be a neutrosophic ring and $P=P_{0}+P_{1} I=\left\{a_{0}+a_{1} I ; a_{0} \in P_{0}, a_{1} \in P_{1}\right\}$.

(a) We say that $\mathrm{P}$ is an $\mathrm{AH}$-ideal if $P_{0}$ and $P_{1}$ are ideals in the ring $\mathrm{R}$.

(b) We say that $\mathrm{P}$ is an AHS-ideal if $P_{0}=P_{1}$.

Definition 2.5: [6]

Let $\left(\mathrm{R}\left(I_{1}, I_{2}\right),+, \times\right)$ be a refined neutrosophic ring, and $P_{0}, P_{1}, P_{2}$ be three ideals in the ring $\mathrm{R}$ then the set $P=\left(P_{0}, P_{1} I_{1}, P_{2} I_{2}\right)=\left\{\left(a, b I_{1}, c I_{2}\right) ; a \in P_{0}, b \in P_{1}, c \in P_{2}\right\}$ is called a refined neutrosophic AH-ideal.

If $P_{0}=P_{1}=P_{2}$ then $\mathrm{P}$ is called a refined neutrosophic AHS-ideal.

\section{Main concepts and discussion}

In this section we introduce our main definitions, results, and we illustrate some examples.

\section{Definition 3.1:}

Let $M(I)=M+M I$ be a strong/weak neutrosophic module, the set $S=P+Q I=\{x+y I ; x \in P, y \in Q\}$, where $\mathrm{P}$ and $\mathrm{Q}$ are submodules of $V$ is called an AH-submodule of $\mathrm{M}(\mathrm{I})$. If $P=Q, \mathrm{~S}$ is called an AHS-submodule of $\mathrm{M}(\mathrm{I})$. 


\section{Example 3.2:}

We have $\mathrm{M}=Z^{2}=Z \times Z$ is a module over $\mathrm{R}, P=<(0,1)>, Q=<(1,0)>$, are two submodules of M. The set $S=P+Q I=\{(0, a)+(b, 0) I ; a, b \in Z\}$ is an AH-submodule of M(I).

The set $L=P+P I=\{(0, a)+(0, b) I\} ; a, b \in Z$ is an AHS-submodule of $\mathrm{M}(\mathrm{I})$.

\section{Theorem 3.3:}

Let $M(I)=M+M I$ be a neutrosophic weak module over the ring R, and let $S=P+Q I$ be an AH-submodule of $M(I)$, then $\mathrm{S}$ is a submodule.

Proof:

Suppose that $x=a+b I, y=c+d I \in S ; a, c \in P, b, d \in Q$,

$x+y=(a+c)+(b+d) I \in S$. For each scalar $m \in R$ we obtain $m \cdot x=m . a+(m \cdot b) I \in S$, since

$P$ and $Q$ are submodules; thus $S=P+Q I$ is a submodule of $\mathrm{M}(\mathrm{I})$ over the ring $\mathrm{R}$.

\section{Theorem 3.4:}

Let $\mathrm{M}(\mathrm{I})$ be a neutrosophic strong module over a neutrosophic ring $\mathrm{R}(\mathrm{I})$, let $S=P+P I$ be an AHS-submodule. Then $\mathrm{S}$ is a submodule of $\mathrm{M}(\mathrm{I})$.

Proof:

Suppose that $x=a+b I, y=c+d I \in S ; a, c, b, c \in P$,

$x+y=(a+c)+(b+d) I \in S$. Let $m=t+d I \in R(I)$ be a neutrosophic scalar, we find

$m \cdot x=(t . a)+(d \cdot a+d \cdot b+t . b) I \in S$, since $d . a+d . b+t . b \in P$, thus we get the desired result.

A strong AH-submodule is not supposed to be a submodule. For examples see [4].

\section{Definition 3.5:}

(a) Let $\mathrm{M}$ and $\mathrm{W}$ be two modules, $L_{M}: M \rightarrow W$ be a homomorphism. The AHS-homomorphism can be defined as follows:

$L: M(I) \rightarrow W(I) ; L(a+b I)=L_{M}(a)+L_{M}(b) I$.

(b) If $S=P+Q I$ is an AH-submodule of $\mathrm{M}(\mathrm{I}), L(S)=L_{M}(P)+L_{M}(Q) I$.

(c) If $S=P+Q I$ is an AH-submodule of $\mathrm{W}(\mathrm{I}), L^{-1}(S)=L_{W}^{-1}(P)+L_{W}^{-1}(Q) I$.

(d) $A H-\operatorname{Ker}(L)=\operatorname{Ker}\left(L_{M}\right)+\operatorname{Ker}\left(L_{M}\right) I=\left\{x+y I ; x, y \in \operatorname{Ker}\left(L_{M}\right)\right\}$.

\section{Theorem 3.6:}

Let $\mathrm{W}(\mathrm{I})$ and $\mathrm{M}(\mathrm{I})$ be two neutrosophic strong/weak modules, and $L: M(I) \rightarrow W(I)$ be an AHS-homomorphism:

(a) $A H-\operatorname{Ker}(L)$ is an AHS-submodule of $\mathrm{M}(\mathrm{I})$. 
(b) If $S=P+Q I$ is an AH-submodule of $\mathrm{M}(\mathrm{I}), L(S)$ is an AH-submodule of $\mathrm{W}(\mathrm{I})$.

(c) If $S=P+Q I$ is an AH-submodule of $\mathrm{W}(\mathrm{I}), L^{-1}(S)$ is an AH-submodule of $\mathrm{M}(\mathrm{I})$.

Proof:

(a) Since $\operatorname{Ker}\left(L_{M}\right)$ is a submodule of M, we find that $A H-\operatorname{Ker}(L)=\operatorname{Ker}\left(L_{M}\right)+\operatorname{Ker}\left(L_{M}\right) I$ is an AHSsubmodule of $\mathrm{M}(\mathrm{I})$.

(b) We have $L(S)=L_{M}(P)+L_{M}(Q) I$; thus $\mathrm{L}(\mathrm{S})$ is an AH-submodule of $\mathrm{W}(\mathrm{I})$, since $L_{M}(P), L_{M}(Q)$ are submodules of W.

(c) By regarding that $L^{-1}(S)=L_{W}^{-1}(P)+L_{W}^{-1}(Q) I, L_{W}^{-1}(P)$ and $L_{W}^{-1}(Q)$ are submodules of $\mathrm{M}$, we obtain that $L^{-1}(S)$ is an AH-subModule of $\mathrm{M}(\mathrm{I})$.

\section{Theorem 3.7:}

Let $\mathrm{W}(\mathrm{I})$ and $\mathrm{M}(\mathrm{I})$ be two neutrosophic strong modules over a neutrosophic ring $\mathrm{R}(\mathrm{I})$, and $L: M(I) \rightarrow W(I)$ be an AHS-homomorphism. Then

$L(x+y)=L(x)+L(y), L(m \cdot x)=m \cdot L(x)$, for all $x, y \in M(I), m \in R(I)$.

Proof:

Suppose $x=a+b I, y=c+d I ; a, b, c, d \in M$, and $m=s+t I \in K(I)$, we have

$L(x+y)=L([a+c]+[b+d] I)=L_{M}(a+c)+L_{M}(b+d) I=\left[L_{M}(a)+L_{M}(b) I\right]+\left[L_{M}(c)+L_{M}(d) I\right]=$ $L(x)+L(y)$.

$m \cdot x=(s \cdot a)+(s \cdot b+t \cdot a+t \cdot b) I, L(m \cdot x)=L_{M}(s \cdot a)+L_{M}(s \cdot b+t \cdot a+t \cdot b) I$

$=s \cdot L_{M}(a)+\left[s \cdot L_{M}(b)+t \cdot L_{M}(a)+t \cdot L_{M}(b)\right] I=(s+t I) \cdot\left(L_{M}(a)+L_{M}(b) I\right)=m \cdot L(x)$.

\section{Theorem 3.8:}

Let $S=P+Q I$ be an AH-submodule of a neutrosophic weak module $\mathrm{M}(\mathrm{I})$ over a ring $\mathrm{R}$, suppose that $X=\left\{x_{i} ; 1 \leq i \leq n\right\}$ is a bases of $\mathrm{P}$ and $Y=\left\{y_{j} ; 1 \leq j \leq m\right\}$ is a bases of $\mathrm{Q}$ then $X \cup Y I$ is a bases of $\mathrm{S}$.

Proof:

Let $z=x+y I$ be an arbitrary element in $\mathrm{S} ; x \in P, y \in Q$. Since $\mathrm{P}$ and $\mathrm{Q}$ are submodules of $\mathrm{M}$ we can write $x=a_{1} x_{1}+a_{2} x_{2}+\cdots+a_{n} x_{n} ; a_{i} \in R$ and $x_{i} \in X, y=b_{1} y_{1}+b_{2} y_{2}+\cdots+b_{m} y_{m} ; b_{i} \in K, y_{i} \in Y$.

Now we obtain $z=\left(a_{1} x_{1}+\cdots+a_{n} x_{n}\right)+\left(b_{1} y_{1} I+\cdots+b_{m} y_{m} I\right)$; thus $X \cup Y I$ generates the subspace $\mathrm{S}$.

$X \cup Y I$ is linearly independent set. Assume that $\left(a_{1} x_{1}+\cdots+a_{n} x_{n}\right)+\left(b_{1} y_{1} I+\cdots+b_{m} y_{m} I\right)=0$, this implies $a_{1} x_{1}+a_{2} x_{2}+\cdots+a_{n} x_{n}=0$ and $\left(b_{1} y_{1}+b_{2} y_{2}+\cdots+b_{m} y_{m}\right) I=0$. Since $\mathrm{X}$ and $\mathrm{Y}$ are linearly independent sets over R, we get $a_{i}=b_{j}=0$ for all $i, j$ and $X \cup Y I$ is linearly independent then it is a basis of S.

\section{Result 3.9:}


Let $S=P+Q I$ be an AH-submodule of a neutrosophic weak module $\mathrm{M}(\mathrm{I})$ with finite dimension over a ring $\mathrm{R}$, from Theorem 3.8 and the fact that $X \cap Y I=\emptyset$, we find $\operatorname{dim}(S)=\operatorname{dim}(P)+\operatorname{dim}(Q)$.

\section{Example 3.10:}

Let $\mathrm{M}=Z^{3}=Z \times Z \times Z$ is a module over the ring $\mathrm{Z}, P=<(0,0,1)>, Q=<(0,1,0)>$ be two submodules of $\mathrm{M}$,

(a) $S=P+Q I=\{(0,0, m)+(0, n, 0) I ; m, n \in Z\}$ is an AH-submodule of M(I).

(b) The set $\{(0,0,1),(0,1,0) I\}$ is a bases of $\mathrm{S}, \operatorname{dim}(S)=\operatorname{dim}(P)+\operatorname{dim}(Q)=1+1=2$.

(c) $L_{M}: M \rightarrow M ; L_{M}(x, y, z)=(x+y, y, z)$ for all $x, y, z \in Z$ is a homomorphism, the corresponding AHShomomorphism is

$L: M(I) \rightarrow M(I) ; L[(x, y, z)+(a, b, c) I]=L_{M}(x, y, z)+L_{M}(a, b, c) I=(x+y, y, z)+(a+b, b, c) I$.

(d) $L(S)=L_{M}(P)+L_{M}(Q)=L_{M}\{(0,0, m)\}+L_{M}\{(0, n, 0)\} I=\{(0,0, m)+(n, n, 0) I\} ; m, n \in Z$, which is an AHsubmodule of $\mathrm{M}(\mathrm{I})$.

\section{Example 3.11 :}

Let $\mathrm{M}=Z^{2}=Z \times Z, \mathrm{~W}=Z^{3}=Z \times Z \times Z$ be two modules over the ring $Z, L_{M}: M \rightarrow W ; L_{M}(x, y)=(x+y, x+$ $y, x+y)$ is a homomorphism. The corresponding AHS-homomorphism is

$L: M(I) \rightarrow W(I) ; L[(x, y)+(a, b) I]=(x+y, x+y, x+y)+(a+b, a+b, a+b) I$.

$\operatorname{Ker} L_{M}=<(1,-1)>, A H-\operatorname{Ker}(L)=\operatorname{Ker}\left(L_{M}\right)+\operatorname{Ker}\left(L_{M}\right) I=<(1,-1)>+<(1,-1)>I=$

$\{(a,-a)+(b,-b) I ; a, b \in Z\}$ which is an AHS-submodule of M(I).

We find $\operatorname{dim}(\operatorname{Ker}(L))=1+1=2$.

\section{Definition 3.12:}

Let $\mathrm{M}(\mathrm{I})$ be a neutrosophic strong/weak module, $S=P+Q I$ be an AH-submodule of $\mathrm{M}(\mathrm{I})$, we define

AH-Quotient module as:

$M(I) / S=M / P+(M / Q) I=(x+P)+(y+Q) I ; x, y \in M$.

\section{Theorem 3.13:}

Let $\mathrm{M}(\mathrm{I})$ be a neutrosophic weak module over a ring $\mathrm{R}$, and $S=P+Q I$ be an AH-submodule of M(I). The AHQuotient $M(I) / S$ is a module with respect to the following operations:

Addition: $[(x+P)+(y+Q) I]+[(a+P)+(b+Q)] I=(x+a+P)+(y+b+Q) I ; x, y, a, b \in M$.

Multiplication by a scalar: $(m) \cdot[(x+P)+(y+Q) I]=(m \cdot x+P)+(m \cdot y+Q) I$;

$x, y \in M$ and $m \in R$.

Proof:

It is easy to check that operations are well defined, and $(M(I) / S,+)$ is abelian group. 
Let $z=[(x+P)+(y+Q) I] \in M(I) / S$, we have 1. $z=z$.

Assume that $m, n \in R$, we have $m \cdot(n \cdot z)=m \cdot[(n \cdot x+P)+(n \cdot y+Q) I]=(m \cdot n \cdot x+P)+(m \cdot n \cdot y+Q) I=$ (m.n).z.

$(m+n) \cdot z=[(m+n) \cdot x+P]+[(m+n) \cdot y+Q] I=m \cdot z+n \cdot z$.

Let $h=[(a+P)+(b+Q) I] \in M(I) / S, z+h=(x+a+P)+(y+b+Q) I$,

$m \cdot(z+h)=(m \cdot x+m \cdot a+P)+(m \cdot y+m \cdot b+Q) m \cdot z+m \cdot h$.

\section{Example 3.14:}

We have $\mathrm{M}=Q^{2}=Q \times Q$ is a module over the ring $\mathrm{Z}, P=<(0,1)>, N=<(1,0)>$ are two submodules of $\mathrm{M}$,

$S=P+N I=\{(0, a)+(b, 0) I ; a, b \in Q\}$ is an AH-subspace of $\mathrm{M}(\mathrm{I})$.

The AH-Quotient is $M(I) / S=\{[(x, y)+P]+[(a, b)+N] I ; x, y, a, b \in Q\}$.

We clarify operations on $M(I) / S$ as follows:

$x=[(2,1)+P]+[(1,3)+N] I, y=[(2,5)+P]+[(1,1)+N] I$ are two elements in $V(I) / S, m=$ 3 is a scalar in $Z$.

$x+y=[(4,6)+P]+[(2,4)+N] I, 3 \cdot x=[(6,3)+P]+[(3,9)+N] I$.

\section{Remark 3.15:}

If $S=P+P I$ is an AHS-submodule of a neutrosophic weak module $\mathrm{M}(\mathrm{I})$ over the ring R, then AH-Quotient $M(I) / S=M / P+M / P I$ is a weak neutrosophic module, since $M / P$ is a module.

We introduce the following result, it determines the algebraic structure of neutrosophic module.

\section{Theorem 3.16:}

Let $(\mathrm{N},+,$.$) be a module over the commutative ring \mathrm{R}, N(I)$ be the corresponding weak neutrosophic module over $\mathrm{R}$. Then

$N(I) \cong N \times N$.

Proof:

Define $f: N \times N \rightarrow N(I) ; f(x, y)=x+y I ; x, y \in N$, it is easy to see that $f$ is well defined bijective map.

Let $(x, y),(z, t) \in M \times M, r \in R$, we have $(x, y)+(z, t)=(x+z, y+t), r .(x, y)=(r \cdot x, r \cdot y)$,

$f[(x, y)+(z, t)]=(x+z)+(y+t) I=(x+y I)+(z+t I)=f(x, y)+f(z, t)$.

$f[r .(x, y)]=r \cdot x+r \cdot y I=r .(x+y I)=r \cdot f(x)$. Hence $f$ is a module isomorphism.

\section{Result 3.17:}

Theorem 3.16 shows that the concept of weak neutrosophic module is a rediscovering of direct product of a module with itself, thus all results in [11] can be obtained easily according to this result. 
According to the previous result, we can find that every submodule of a weak neutrosophic module is an AHsubmodule, since every submodule of $M \times M$ has the form $W \times N ; W, N$ are submodules of $\mathrm{M}$.

\section{Conclusion}

In this article, we have defined the concepts of AH-submodule, AHS-submodule, and AHS-homomorphism in neutrosophic module as new generalizaion of AH-substructures in neutrosophic vector spaces. Also, we have studied some basic properties of these concepts.

On the other hand, we have proved that every weak neutrosophic module $\mathrm{M}(\mathrm{I})$ over any commutative ring $\mathrm{R}$ is isomorphic to the direct product of $\mathrm{M}$ with itself.

\section{Future Aspect}

This study can be extended into more neutrosophic structures.

Funding: "This research received no external funding"

Conflicts of Interest: "The authors declare no conflict of interest."

\section{References}

[1] Abdel-Basset, M., Mai M., Mohamed E., Francisco C., and Abd El-Nasser, H. Z., "Cosine Similarity Measures of Bipolar Neutrosophic Set for Diagnosis of Bipolar Disorder Diseases", Artificial Intelligence in Medicine $101,2019,101735$.

[2] Abdel-Basset, M., Mohamed E., Abduallah G., and Florentin S., "A Novel Model for Evaluation Hospital Medical Care Systems Based on Plithogenic Sets" Artificial Intelligence in Medicine 100 2019, 101710.

[3] Abdel-Basset, M., Gunasekaran M., Abduallah G., and Victor C., "A Novel Intelligent Medical Decision Support Model Based on Soft Computing and IoT", IEEE Internet of Things Journal , 2019.

[4] Abdel-Basset, M., Abduallah G., Gunasekaran M., and Hoang Viet L., "A Novel Group Decision Making Model Based on Neutrosophic Sets for Heart Disease Diagnosis", Multimedia Tools and Applications , 1-26, 2019.

[5] Abobala, M., "On Some Special Substructures of Neutrosophic Rings and Their Properties", International Journal of Neutrosophic Science", Vol. 4 , pp.72-81, 2020.

[6] Abobala, M., "On Some Special Substructures of Refined Neutrosophic Rings", International Journal of Neutrosophic Science, Vol. 5, pp.59-66, 2020.

[7] Abobala, M., "Classical Homomorphisms Between Refined Neutrosophic Rings and Neutrosophic Rings", International Journal of Neutrosophic Science, Vol. 5, pp.72-75, 2020.

[8] Abobala, M., " AH-Subspaces in Neutrosohic Vector Spaces", International Journal of Neutrosophic Science, Vol. 6, PP. 80-86, 2020.

[9] Adeleke, E.O., Agboola, A.A.A., and Smarandache, F., "Refined Neutrosophic Rings I", International Journal of Neutrosophic Science, Vol. 2 , pp.77-81, 2020.

[10] Agboola, A.A.A., and Akinleye, S.A., "Neutrosophic Vector Spaces", Neutrosophic Sets and Systems, Vol. 4 , pp. 9-17, 2014.

[11] Agboola, A.A.A., Akwu, A.D., and Oyebo, Y.T., "Neutrosophic Groups and Subgroups", International .J .Math.Combin, Vol. 3, pp. 1-9, 2012. 
[12] Agboola, A.A.A., Akinola, A.D, and Oyebola, O.Y.," NeutrosophicRings I ", International J.Mathcombin, Vol. 4, pp. 1-14, 2011.

[13] Injrou, S., "Linear Algebra 2 ", Tishreen University Press, pp.180-230, 2015.

[14] Kandasamy, V.W.B., and Smarandache, F., "Some Neutrosophic Algebraic Structures and Neutrosophic NAlgebraic Structures", Hexis, Phonex, Arizona 2006.

[15] Olgan, N., and Khatib, A., "Neutrosophic Modules", Journal of Biostatistic and Biometric Application", Vol. 3, pp.1-16, 2018. 